\title{
Loss of psoas shadow in acute abdomen: An often missed sign in X-rays
}

\author{
Min-Hsuan Yen and Cheng-Maw Ho* \\ Department of Surgery, National Taiwan University Hospital and National Taiwan University College of Medicine, Taipei, Taiwan
}

Computed Tomography (CT) is widely used as a diagnostic tool for identification of acute intra-abdominal pathology. Plain X-ray examination provides, although limited, sometimes important information. Herein, we demonstrated a typical image of iliopsoas muscle abscess as the loss of psoas shadow in plain abdominal X-ray.

\section{Case description}

A 28-year-old woman without underlying disease came to our emergency room because of fever and abdominal pain in the right lower quadrant for 4 days. The pain made her unable to fully extend her leg and difficult walking (limp sign). Her white blood cell count was $18100 / \mu \mathrm{L}$ ( $83 \%$ neutrophils). Kidney-ureter-bladder radiography
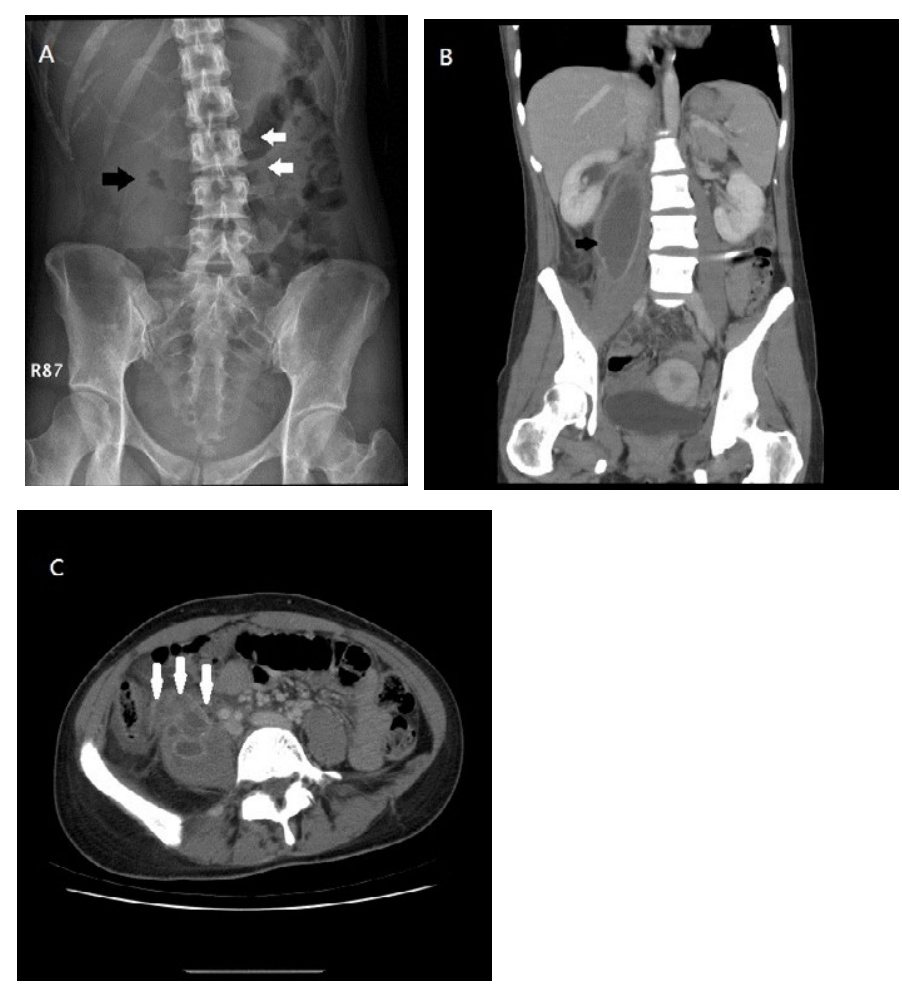

Figure 1. Loss of psoas shadow signs in roenterology. A. Kidney-ureter-bladder radiography revealed the loss of margin of the right psoas muscle (black arrow). B. corresponding right psoas muscle abscess shown in coronal view of contrast-enhanced computed tomography (black arrow).C. perforated appendicitis into the retroperitoneum (white arrows).

Copyright: $@ 2015$ Yen MH. This is an open-access article distributed under the terms of the Creative Commons Attribution License, which permits unrestricted use, distribution, and reproduction in any medium, provided the original author and source are credited. revealed the loss of margin of the right psoas muscle (Figure 1A, black arrow). Contrast-enhanced $\mathrm{CT}$ of the abdomen confirmed the right psoas muscle abscess (Figure 1B, black arrow), which was caused by perforated appendicitis into the retroperitoneum (Figure 1C). CTguided catheter drainage of the right psoas yielded $120 \mathrm{ml}$ of purulent fluid and decompressed the abscess; the fluid yielded Bacteroides uniformis. The catheter was positioned until the drainage fluid was almost dry. Antibiotic therapy for appendicitis was continued for 6 weeks. Follow-up CT images revealed complete resolution of the psoas abscess and appendicitis.

\section{Discussion}

The typical psoas sign in plain abdominal X-ray (loss of psoas shadow) and the classic triad of pain, fever, and limp (first described by Mynter [1]), as demonstrated in our case, are rarely seen. Continuous spread from gastrointestinal tract infection was being recognized as the major cause of the psoas abscess, and CT was used as the diagnostic modality of choice [2]. Management included percutaneous drainage, the administration of appropriate antibiotics, and critical surgical consideration of primary source control.

Psoas shadow is the usual finding in plain abdominal X-ray. The absence of psoas shadow indicated the inflammation around the psoas muscle, and warrants further evaluation.

\section{Authors' contributions}

Study concept and design (HCM), acquisition of subjects and/or data (YMH), and preparation of manuscript (HCM, YMH)

\section{References}

1. Mynter H (1881) Acutepsoitis. Buffalo Med Surg J 21:202-210.

2. Tabrizian P, Nguyen SQ, Greenstein A, Rajhbeharry Singh U, Divino CM (2009) Management and treatment of iliopsoas abscess. Arch Surg 144: 946-949. [Crossref]

Correspondence to: Cheng-Maw Ho, Department of Surgery, National Taiwan University Hospital and National Taiwan University College of Medicine, Taipei, Taiwan, Tel: +886-2-23123456, ext. 65914, Fax: +886-2-2356-8810; E-mail: miningho@ntu.edu.tw

Received: May 30, 2015; Accepted: July 11, 2015; Published: July 14, 2015 